\title{
Heart failure treatment according to the 2021 European Society of Cardiology Guidelines - experiences with SGLT2 inhibitors have changed the treatment strategy
}

Previously the treatment with $\beta$-blockers, angiotensin-converting enzyme inhibitors or angiotensin receptor blockers and mineralocorticoid receptor antagonists, was shown to provide benefits in terms of mortality and morbidity in heart failure (HF) patients with reduced ejection fraction (HFrEF) [1]. Later substantial improvement in outcomes with the angiotensin receptor-neprilysin inhibitor (sacubitril-valsartan) above the benefits provided by the angiotensin-converting enzyme inhibitor enalapril was shown [2]. Recently several clinical trials unexpectedly showed favorable impact on cardiovascular outcomes of some sodium-glucose co-transporter 2 inhibitors (SGLT2-I), namely: canagliflozin, dapagliflozin, and empagliflozin [3-8]. The positive cardiovascular effects of SGLT2 inhibitors became apparent within months from the beginning of treatment, suggesting that the mechanisms beyond improved glucose control and reduced atherosclerosis are involved in cardiovascular risk reduction [3 8].

In the Study to Evaluate the Effect of Dapagliflozin on the Incidence of Worsening Heart Failure or Cardiovascular Death in Patients with Chronic Heart Failure (DAPA-HF) 4744 patients with heart failure (NYHA II-IV) with reduced ejection fraction $(<40 \%)$ with diabetes $(45 \%)$ or without $(55 \%)$ were randomized to receive dapagliflozin $10 \mathrm{mg} /$ day or placebo, on top of optimal standard therapy for heart failure. A significant reduction in the primary outcomes, defined as a composite of worsening heart failure or cardiovascular death, were achieved in patients receiving dapagliflozin (HR 0.74; $95 \% \mathrm{Cl}, 0.65$ to $0.85 ; \mathrm{p}<0.001$ ) [7].

In the Empagliflozin Outcome Trial in Patients with Chronic Heart Failure and a Reduced Ejection Fraction (EMPEROR-Reduced) 3730 patients with heart failure (NYHA II-IV) with reduced ejection fraction (<40\%) with diabetes $(50 \%)$ or without $(50 \%)$ were randomized to receive empagliflozin $10 \mathrm{mg} /$ day or placebo, on top of optimal standard therapy for heart failure. The primary outcomes, defined as a composite of cardiovascular death or hospitalization for worsening heart failure, were significantly reduced in the empagliflozin arm (HR 0.75; 95\% Cl, 0.65 to 0.86; $p<0.001$ ) [8].

The meta-analysis of these two large-scale trials including 8474 patients demonstrated a $13 \%$ reduction in all-cause death (HR 0.87, 95\% Cl 0.77-0.98; $\mathrm{p}=0.018$ ) and a $14 \%$ reduction in cardiovascular death (HR 0.86 , 95\% Cl 0.76-0.98; $p=0.027$ ) [9]. Moreover, a $26 \%$ reduction in the combined risk of cardiovascular death or first hospitalization for heart failure (HR $0.74,95 \% \mathrm{Cl}$ $0.68-0.82 ; p<0.0001$ ) was observed in the patients treated with dapagliflozin or empagliflozin versus placebo [9].

After the publication of the DAPA-HF and the EMPEROR-Reduced trial results both showing the exceptional clinical benefits of dapagliflozin and empagliflozin respectively, applied on top of guidelines-recommended therapy for the treatment of patients with chronic HFrEF, regardless of coexistence of diabetes mellitus, some changes in ESC guidelines were expected [7, 8].

Corresponding author: Jacek Kubica, Collegium Medicum, Nicolaus Copernicus University, Bydgoszcz, Poland, e-mail: jkubica@cm.umk.pl Medical Research Journal 2021; Volume 6, Number 3, 163-165, 10.5603/MRJ.2021.0046, Copyright @ 2021 Via Medica, ISSN 2451-2591, e-ISSN 2451-4101

This article is available in open access under Creative Common Attribution-Non-Commercial-No Derivatives 4.0 International (CC BY-NC-ND 4.0) license, allowing to download articles and share them with others as long as they credit the authors and the publisher, but without permission to change them in any way or use them commercially. 
Indeed, as expected, the recent ESC guidelines for the diagnosis and treatment of acute and chronic heart failure contain several changes that may be considered revolutionary [10].

To achieve three major goals of treatment defined as reduction in mortality, prevention of recurrent hospitalizations due to worsening HF, and improvement in clinical status, functional capacity, and quality of life, a new simplified treatment algorithm for HFrEF, according to phenotypes, has been introduced [10].

The cornerstone therapy with angiotensin-converting enzyme inhibitors (ACE-I) or an angiotensin receptor-neprilysin inhibitor (ARNI), beta-blockers $(\mathrm{BB})$, and mineralocorticoid receptor antagonists (MRA) is recommended for patients with HFrEF unless the drugs are contraindicated or not tolerated. ACE-I should be replaced with $A R N I$ in patients who remain symptomatic on ACE-I, beta-blocker, and MRA; however, ARNI may be also applied instead of an ACE-I as first-line therapy. Angiotensin-receptor blockers (ARBs) should be used in patients who are intolerant to ACE-I or ARNI. SGLT2-I - dapagliflozin or empagliflozin should be used on top of this cornerstone therapy in all patients with HFrEF unless contraindicated or not tolerated (class I of recommendations) [10].

The recommended four-component (ACE-I/ /ARNI + BB + MRA + SGLT2-I) first-line therapy has been proved to reduce the risk of HF hospitalization and death [7-9].

Additionally, according to the results of the Vericiguat Global Study in Subjects with Heart Failure with Reduced Ejection Fraction (VICTORIA) the oral soluble guanylate cyclase receptor stimulator, vericiguat, may be considered in patients in NYHA class II-IV who have had worsening HF despite treatment with three-component cornerstone therapy (without SGLT2-I) to reduce the risk of CV mortality or HF hospitalization (class IIb of recommendations) $[10,11]$.

The great emphasis in these guidelines was placed on prevention, including programs to improve outcomes, influenza and pneumococcal vaccinations, cardiac rehabilitation programs, and home telemonitoring in patients with heart failure and preserved ejection fraction [10].

Recommendations for the management of patients after hospitalization for HF highlights the need for adequate preparation for discharge from the hospital and cooperation with HF patients after discharge to improve adherence to treatment [10]. These guidelines create space for the use of already known and used tools and methods, including scales and self-reported questionnaires [11-21].

Summing up, the 2021 European Society of Cardiology Guidelines present a completely new strategy for the treatment of HF patients that offers a possibility to improve clinical outcomes and achieve long-term clinical improvement much faster than previously possible. The SGLT2-Is - dapagliflozin and empagliflozin play a key role in the new strategy.

\section{References}

1. Felker GM. Building the Foundation for a New Era of Quadruple Therapy in Heart Failure. Circulation. 2020; 141(2): 112-114, doi: 10.1161/CIRCULATIONAHA.119.044578, indexed in Pubmed: 31736333.

2. McMurray JJV, Packer M, Desai AS, et al. PARADIGM-HF Investigators and Committees. Angiotensin-neprilysin inhibition versus enalapril in heart failure. N Engl J Med. 2014; 371 (11): 993-1004, doi: 10.1056/NEJMoa1409077, indexed in Pubmed: 25176015.

3. Zinman B, Wanner C, Lachin JM, et al. EMPA-REG OUTCOME Investigators. Empagliflozin, Cardiovascular Outcomes, and Mortality in Type 2 Diabetes. N Engl J Med. 2015; 373(22): 2117-2128, doi: 10.1056/NEJMoa1504720, indexed in Pubmed: 26378978

4. Neal B, Perkovic V, Mahaffey KW, et al. CANVAS Program Collaborative Group. Canagliflozin and Cardiovascular and Renal Events in Type 2 Diabetes. N Engl J Med. 2017; 377(7): 644-657, doi: 10.1056/NEJMoa1611925, indexed in Pubmed: 28605608

5. Wiviott SD, Raz I, Bonaca MP, et al. DECLARE-TIMI 58 Investigators. Dapagliflozin and Cardiovascular Outcomes in Type 2 Diabetes. N Engl J Med. 2019; 380(4): 347-357, doi: 10.1056/NEJMoa1812389, indexed in Pubmed: 30415602

6. Perkovic V, Jardine MJ, Neal B, et al. CREDENCE Trial Investigators. Canagliflozin and Renal Outcomes in Type 2 Diabetes and Nephropathy. N Engl J Med. 2019; 380(24): 2295-2306, doi: 10.1056/NEJMoa1811744, indexed in Pubmed: 30990260.

7. McMurray JJV, Solomon SD, Inzucchi SE, et al. DAPA-HF Trial Committees and Investigators. Dapagliflozin in Patients with Heart Failure and Reduced Ejection Fraction. N Engl J Med. 2019; 381(21): 1995-2008, doi: 10.1056/NEJMoa1911303, indexed in Pubmed: 31535829

8. Packer M, Anker SD, Butler J, et al. EMPEROR-Reduced Trial Investigators. Cardiovascular and Renal Outcomes with Empagliflozin in Heart Failure. N Engl J Med. 2020; 383(15): 1413-1424, doi: 10.1056/NEJMoa2022190, indexed in Pubmed: 32865377.

9. Zannad F, Ferreira JP, Pocock SJ, et al. SGLT2 inhibitors in patients with heart failure with reduced ejection fraction: a meta-analysis of the EMPEROR-Reduced and DAPA-HF trials. Lancet. 2020; 396(10254): 819-829, doi: 10.1016/S0140-6736(20)31824-9, indexed in Pubmed: 32877652 .

10. McDonagh TA, Metra M, Adamo M et al. ESC Scientific Document Group . 2021 ESC Guidelines for the diagnosis and treatment of acute and chronic heart failure. Eur Heart J. 2021 [Epub ahead of print], doi: 10.1093/eurheartj/ehab368, indexed in Pubmed: 34447992

11. Armstrong PW, Pieske B, Anstrom KJ, et al. VICTORIA Study Group. Vericiguat in Patients with Heart Failure and Reduced Ejection Fraction. N Engl J Med. 2020; 382(20): 1883-1893, doi: 10.1056/NEJMoa1915928, indexed in Pubmed: 32222134

12. Buszko K, Obońska K, Michalski P, et al. The Adherence Scale in Chronic Diseases (ASCD). The power of knowledge: the key to successful patient — health care provider cooperation. Medical Research Journal. 2016; 1(1): 37-42, doi: 10.5603/mrj.2016.0006.

13. Kubica A, Kosobucka A, Michalski P, et al. The Adherence in Chronic Diseases Scale - a new tool to monitor implementation of a treatment plan. Folia Cardiol. 2017; 12: 19-26, doi: 10.5603/FC.2016.0000.

14. Kubica A, Pietrzykowski $Ł$. The therapeutic plan implementation in patients discharged from the hospital after myocardial infarction. Medical Research Journal. 2021; 6(2): 79-82, doi: 10.5603/mrj.a2021.0024.

15. Kosobucka A, Pietrzykowski $Ł$, Michalski P, et al. Impact of readiness for discharge from the hospital on the implementation of the therapeutic plan. Medical Research Journal. 2020; 5(4): 256-264, doi: 10.5603/mrj.a2020.0047

16. Kubica A, Obońska K, Fabiszak T, et al. Adherence to antiplatelet treatment with P2Y12 receptor inhibitors. Is there anything we can do to improve it? A systematic review of randomized trials. Curr Med Res Opin. 2016; 32(8): 1441-1451, doi: 10.1080/03007995.2016.1182901, indexed in Pubmed: 27112628.

17. Kubica A, Gruchała M, Jaguszewski M, et al. Adherence to treatment - a pivotal issue in long-term treatment of patients with cardiovascular diseases. An expert standpoint. Medical Research Journal. 2018; 2(4): 123-127, doi: 10.5603/mrj.2017.0016. 
18. Kubica A. Self-reported questionnaires for a comprehensive assessment of patients after acute coronary syndrome. Medical Research Journal. 2019; 4(2): 106-109, doi: 10.5603/mrj.a2019.0021.

19. Kubica A, Kosobucka A, Michalski P, et al. Self-reported questionnaires for assessment adherence to treatment in patients with cardiovascular diseases. Medical Research Journal. 2018; 2(4): 115-122, doi 10.5603/mrj.2017.0015
20. Buszko K, Pietrzykowski Ł, Michalski P, et al. Validation of the Functioning in Chronic Illness Scale (FCIS). Medical Research Journal. 2018; 3(2): 63-69, doi: 10.5603/mrj.2018.0011.

21. Buszko K, Kosobucka A, Michalski $P$ et al. The readiness for hospita discharge of patients after acute myocardial infarction: a new self-reported questionnaire. Medical Research Journal. 2017; 2(1): 20-28, doi: 10.5603/mrj.2017.0004. 